\title{
Brain MR image normalization in texture analysis of multiple sclerosis
}

\author{
C. P. Loizou, M. Pantziaris, C. S. Pattichis, I. Seimenis \\ 1. Department of Computer Science, Intercollege, Limassol, Cyprus. 2. Cyprus Institute of Neurology and Genetics, \\ Nicosia, Cyprus. 3. Department of Computer Science, University of Cyprus, Nicosia, Cyprus. 4. Medical Diagnostic Center \\ "Ayios Therissos", Nicosia, Cyprus.
}

Correspondence: Christos P. Loizou. Address: Department of Computer Science, Intercollege, P. O. Box 51604, CY-3507 Limassol, Cyprus. E-mail: panloicy@logosnet.cy.net; loizou.c@lim.intercollege.ac.cy.

Received: March 30, 2012

DOI : $10.5430 / j b g c . v 3 n 1 p 20$
Accepted: May 31, 2012

URL: http://dx.doi.org/10.5430/jbgc.v3n1p20

\section{Abstract}

A problem that occurs in quantitative texture analysis of magnetic resonance imaging (MRI) is that there are intra-scan and inter-scan image intensity variations due to the MRI instrumentation. Therefore, image intensity normalization methods should be applied prior to further image analysis. The objective of this work was to investigate six previously described MRI intensity normalization methods and propose the most appropriate for the pre-processing of brain T2-weighted MR images acquired from 38 symptomatic untreated multiple sclerosis (MS) subjects. The following normalization methods were investigated: Contrast Stretch Normalization (CSN), Intensity Scaling (IS), Histogram Stretching (HS), Histogram Normalization (HN), Gaussian Kernel Normalization (GKN), and Histogram Equalization (HE). The main findings of this study can be summarized as follows: 1) Lesion texture features were affected differently by the normalization process for both the 0 and 6-12 months MRI scans. For example, for the features median and contrast there was significant difference between 0 and 6-12 months for the original MRI images but not for the HN normalized ones. On the other hand for the feature complexity there was no significant difference between 0 and 6-12 months for the original MRI images, but there was for the HN normalized images. 2) The statistical lesion feature analysis between the original and the normalized images showed that the $\mathrm{HN}$ method gave the highest number of significant features after normalization for both the 0 and 6-12 months MRI scans. 3) The HN normalization method gave the best performance compared to the other normalization methods with respect to the distance measures, structural similarity index, coefficient of variation, and correlation coefficient between the original and the normalized 0 and 6-12 months MRI scans. Thus, based on the above findings it is recommended that the simple $\mathrm{HN}$ normalization method could be used prior to quantitative texture analysis in the case study presented. The findings of this study provide evidence that texture features of MRI-detectable brain white matter lesions may have an additional potential role in the clinical evaluation of MRI images in MS. This is a prerequisite step in the assessment of texture features as surrogate markers of disease progression. However, a larger scale study is needed to establish the application in clinical practice and for computing shape parameters and texture features that may provide information for better and earlier differentiation between normal tissue and MS lesions.

\section{Key words}

Magnetic resonance imaging, Multiple sclerosis, Image normalization, Texture analysis 


\section{I ntroduction}

Texture analysis is used widely in MRI enabling disease characterization and quantification of disease distribution. Texture features quantify macroscopic lesions and characterize macroscopic changes that may be undetectable using conventional measures of lesion volume and number ${ }^{[11]}$. Furthermore, quantitative texture analysis may provide information that is not easily visible to the human eye. However, texture features are sensitive to the acquisition conditions including magnetic resonance (MR) protocols, MR scanners and MR adjustments ${ }^{[33]}$. This yields unwanted intensity variations which may affect the results of image analysis. An intensity normalization correction stage is therefore essential. It was shown ${ }^{[26,33]}$ that normalization methods improve image compatibility by reducing the variability introduced by different gain settings, different operators, different equipment, and facilitates MR image comparability.

Different approaches to the above problem have been proposed in the literature. The influence of normalization was studied for different acquisition protocols ${ }^{[7]}$. Meier et al. ${ }^{[25]}$ proposed an intra- and an inter-scan normalization method for processing serial MRI scans for direct quantitative evaluation. A histogram mapping was proposed ${ }^{[13]}$, whereas a contrast stretch normalization method based on the maximum and minimum gray scale values in the image was introduced ${ }^{[18]}$. A normalization method for brain MRI using histogram even-order derivative analysis was proposed ${ }^{[5]}$, whereas, a histogram matching method to correct the variations due to scanner sensitivity was presented ${ }^{[35]}$. Finally, a normalization method was proposed, where the original image histogram is stretched and shifted in order to cover all the available gray levels of the image ${ }^{[26]}$.

The objective of our study was to implement and investigate six different normalization methods for brain MR images and propose the most appropriate one for the texture analysis of multiple sclerosis (MS) brain lesions. Preliminary results of this study were also published as a conference paper ${ }^{[22]}$, where only 22 subjects were used for the analysis and the evaluation of the method.

Diagnostic evaluation of MS is generally based on MRI imaging and on clinical signs and symptoms ${ }^{[24,31]}$. Texture features quantify lesion characterization, and several studies have been published for the assessment of MS lesions in: (i) differentiating between lesions for normal white matter (NWM), and the normal appearing white matter (NAWM) ${ }^{[15,39]}$, and (ii) monitoring the progression of the disease ${ }^{[7,10,16,25]}$. It is anticipated that selecting the most appropriate image normalization method will help us in differentiating between normal and abnormal tissue, as well as facilitate disease monitoring.

The layout of the paper is as follows: Section II presents the materials and methods, section III highlights the results of the study, section IV presents the discussion, and section V the concluding remarks.

\section{Materials and methods}

\subsection{Study group and MRI acquisition}

Thirty eight subjects (17 males, and 21 females), aged 34.1 \pm 10.5 (mean age \pm standard deviation) with a clinical isolated syndrome (CIS) of MS and MRI detectable brain lesions were scanned twice at $1.5 \mathrm{~T}$ with an interval of 6-12 months. The transverse MR images used for analysis were obtained using a T2-weighted turbo spin echo pulse sequence (repetition time $=4408 \mathrm{~ms}$, echo time $=100 \mathrm{~ms}$, echo spacing $=10.8 \mathrm{~ms}$ ). The reconstructed image had a slice thickness of $5 \mathrm{~mm}$ and a field of view of $230 \mathrm{~mm}$ with a pixel resolution of 2.226 pixels per $\mathrm{mm}$. Standardized planning procedures were followed during each MRI examination. The MRI images were acquired using a $1.5 \mathrm{~T}$ whole body Philips ACS NT MR imager (Philips Medical Systems, Best, the Netherlands). A built-in quadrature radiofrequency (RF) body coil and a quadrature RF head coil were used for proton excitation and signal detection respectively. 
Initial clinical evaluation was made by an experienced MS neurologist (co-author, M. Pantziaris) who referred subjects for a baseline MRI upon diagnosis and clinically followed all subjects for over five years. All subjects were subjected to an expanded disability status scale (EDSS) test two years after initial diagnosis to quantify disability and then again in five years. They were clinically followed and examined by the MS neurologist following the initial MRI (time 0) and also at two years later. At the initial scan, the stage of the disease was evaluated using the EDSS score ${ }^{[2]}$. This gave starting EDSS scores with a mean of 2.2 and a standard deviation of 0.8 . The number of subjects with EDSS $\leqslant 2$, and EDSS $>2$, two years after the first examination were 23 and 15 respectively. In five years' time the EDSS score gave a mean value of 2.85 and a standard deviation of 1.5 .

Additionally, brain images from 20 healthy, age-matched (mean \pm SD: $30.8 \pm 7.6$ ) volunteers ( 8 males, and 12 females) were carried out to allow segmentation and analysis of normal brain white matter.

\subsection{Manual delineations and visual perception}

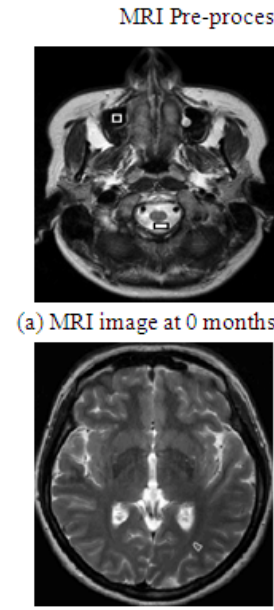

(b) Lesion delineation

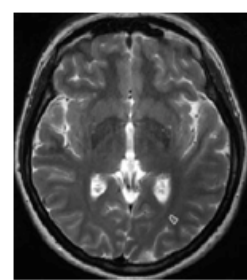

(c) Normalized image with the HN method

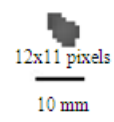

(d) Extracted lesion at 0 months

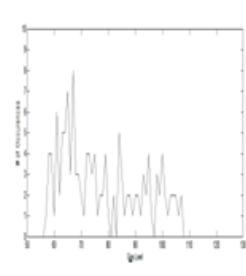

(e) HN normalised histogram

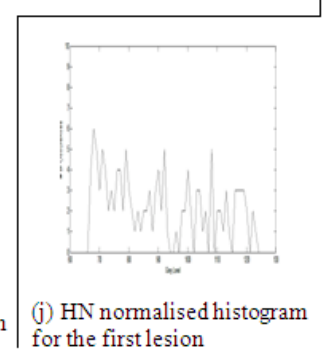

MRI Pre-processing steps at 6-12 months

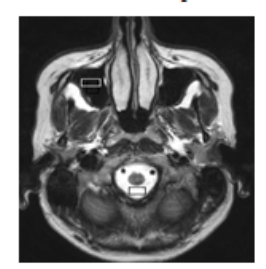

(f) MRI image at 6-12 months

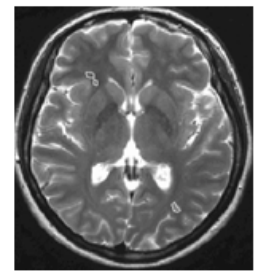

(g) Lesion delineation

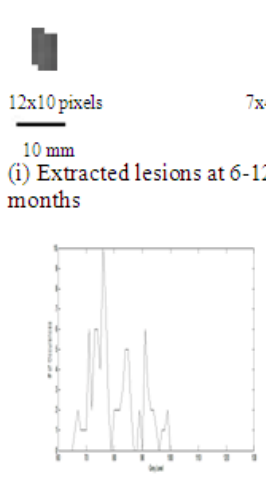

(k) HN normalised histogram for the second lesion

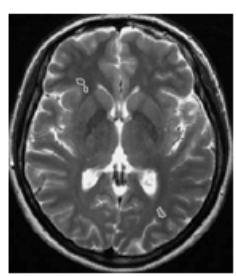

(h) Normalized image with the HN method
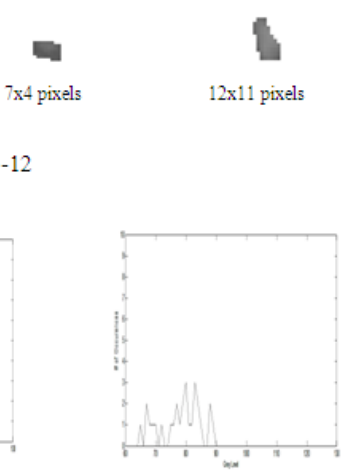

(1) HN normalised histogram for the third lesion

Figure 1. An example of MRI preprocessing steps of a 34 year old female patient at 0 and 6-12 months. (a) Selection of the BWM and GWM from the first slide of the whole MRI session at 0 months (EDSS=1, BWM=5, GWM=249), (b) MRI image before normalization with lesion delineation, (c) MRI normalized image from (b) with the HN method, (d) extracted lesion at 0 months (median (IQR): 92 (11)) resized by a factor of 3, (e) histogram of the lesion at 0 months (median (IQR): 86(52)), (f) selection of the BWM and GWM from the first slide at 6-12 months (EDSS=2, BWM=6, GWM=245), (g) MRI image at 6-12 months before normalization with lesions delineation, (h) MRI normalized image from (g) with the HN method, (i) extracted lesions at 6-12 months (median (IQR): 123 (13), 96 (11), 98 (11)) resized by a factor of 3, (j-1) histograms of the 3 lesions at 6-12 months after image normalization (median (IQR): 98 (55), 83 (31), 78 (22)) with the HN method. 
All MRI-detectable brain lesions were identified and segmented by an experienced MS neurologist and confirmed by a radiologist. Only well-defined areas of hyperintensity on T2-weighted MR images were considered as MS plaques. The neurologist manually delineated (using the mouse) the brain lesions by selecting consecutive points at the visually defined borders between the lesions and the adjacent NAWM on the acquired transverse T2-weigted sections. Similar regions corresponding to NAWM were delineated contralaterally to the detected MS lesions. The manual delineations were performed using a graphical user interface implemented in Matlab ${ }^{\circledR}$ developed by our group. For each brain MRI scan of MS subjects, 10 discrete round regions of interest (ROIs) with an approximate radius of 25 pixels were also drawn in brain white matter, usually on the contralateral to the lesion side, to represent NAWM. Every effort was made to avoid white matter areas with subtle, patchy and diffuse abnormal signal intensities. Finally, the neurologist manually segmented cerebrovascular fluid (CSF) areas as well as areas with air (sinuses) from all MS brain scans. Similarly, ROIs representing NWM, CSF and air from the sinuses were arbitrarily segmented from the brain scans of the 20 healthy subjects. Manual segmentation by the MS expert was performed in a blinded manner, without the possibility of identifying the subject, the time-point of the exam or the clinical findings. The selected points and delineations were saved to be used for texture analysis. Figure 1 presents an example of manual selection of the black-white matter (BWM, which is the darkest image area) and gray-white matter (GWM, which is the brightest image area), where the neurologist selects BWM and GWM from the first slide of the whole MRI session at 0 months (see Fig. 1 (a), BWM=5, GWM=249) and at 6-12 months (see Fig. 1 (g), BWM=6, GWM=245), respectively.

\subsection{Interscan intensity normalization}

A normalization algorithm adjusts distributions of each follow up scan to match those of the chosen baseline scan in order to improve image compatibility and facilitate MR image comparability between serial MRI scans ${ }^{[25]}$, such as those obtained from the MS group of this work. In order to select the most appropriate normalization algorithm suitable for MRI brain lesions normalization of MS patients, the following MRI intra-scan intensity normalization methods were implemented and evaluated. An example of interscan intensity normalization method is given in Fig. 1, for 0 and 6-12 months respectively, whereas Fig. 2 illustrates an example of the original and normalized image histograms for all different interscan intensity normalization methods that will be presented below.

1) Contrast Stretch and Normalization $(\mathrm{CSN})^{[18]}$ : The global maximum $\left(g_{\max }\right)$ and minimum $\left(g_{\min }\right)$ of the original image are firstly estimated by excluding the image background which is normally 0 . A $9 \times 9$ pixel window is used in the neighborhood of $g_{\max }$ and $g_{\min }$ to compute the $g_{h i_{-} \text {thres }}$ and $g_{\text {low_thres }}$ respectively by averaging all pixels in the corresponding windows of the original image. That is grayscale values of the original image, above $g_{\max }$ are equated to $g_{\text {hi_thres }}$ and values below $g_{\min }$ are equated to $g_{\text {low_thres }}$, as follows (see also Fig. 2(d)):

$$
f(x, y)=\left(g(x, y)-g_{\text {low_thres }}\right) \cdot \frac{g_{\max }-g_{\min }}{g_{h i \_t h r e s h}-g_{\text {low_thresh }}}+g_{\min }
$$

2) Intensity Scaling (IS): The neurologist selects homogeneous high intensity regions of interest (cerebrospinal fluid or High Intensity Region-GWM) and low intensity regions (air from the sinuses or Low Intensity Region-BWM) from the brain. The image intensities are mapped to the values between GWM and BWM. This was implemented using the Matlab ${ }^{\circledR}$ function gscale as follows (see also Fig. 2(e)):

$$
f(x, y)=\frac{g(x, y)-B W M}{G W M-B W M}
$$

3) Histogram Stretching (HS): The following normalization or contrast stretching transformation for increasing the dynamic range of the resulting image was carried out (see also Fig. 2(f)): 


$$
f(x, y)=\frac{g(x, y)-g_{\min }}{g_{\max }-g_{\min }}
$$

where $g(x, y)$ is the original image gray scale value at coordinates $\mathrm{x}$ and $\mathrm{y}, g_{\max }$ and $g_{\min }$ represent the maximum and the minimum gray scale values in the original image respectively, and $f(x, y)$ the resulted normalized pixel.

4) Histogram Normalization $(\mathrm{HN})^{[26]}$ : The original image histogram is stretched, and shifted in order to cover all the gray scale levels in the image as follows ${ }^{[22,26]}$ (see also Fig. 1 and Fig. 2(g)):

$$
f(x, y)=\frac{G W M-B W M}{g_{\max }-g_{\min }} \cdot\left(g(x, y)-g_{\min }\right)+B W M
$$

If the original histogram of the initial image $g(x, y)$, starts at $g_{\min }$ and extends up to $g_{\max }$ brightness levels, then we can scale up the image so that the pixels in the new image, $f(x, y)$, lie between a minimum level (BWM ) and a maximum level (GWM ). This is done by scaling up the intensity levels according to (4).

5) Gaussian Kernel Normalization $(\mathrm{GKN}){ }^{[17]}$ : A local normalization algorithm is used that normalizes the local mean and variance of the image estimated by a Gaussian Kernel using smoothing operators. The resulted image is as follows (see also Fig. 2(h)):

$$
f(x, y)=\frac{g(x, y)-m_{g}}{\sigma_{g}}
$$

where $m_{g}$ and $\sigma_{g}$ represent the mean and standard deviation of the original image respectively.

6) Histogram Equalization (HE) ${ }^{[13]}$ : Image histogram equalization was carried out using the Matlab ${ }^{\circledR}$ function histeq (see also Fig. 2(i)). Redistribution of the pixels in the image is based on the normalized cumulative histogram, defined as:

$$
H(j)=\frac{1}{M N} \sum_{i=0}^{j} h(i) \quad j=0,1, \ldots P-1
$$

where the histogram of the image $g(x, y)$, is defined as $h(i)$ with 0 to P-1 grey levels. The normalized cumulative histogram can be used as a mapping between the original gray levels in the image and the new gray levels required for enhancement. The enhanced image $f(x, y)$ will have a maximally uniform histogram ${ }^{[29]}$ if it is defined as:

$$
f(x, y)=(P-1) H(g(x, y))
$$

\subsection{Feature extraction: Shape and texture}

In this study, shape parameters and texture features were extracted from all MS lesions detected and segmented, as well as from all the segmented ROIs from the healthy brain areas. The overall shape parameters and texture features for each subject were then estimated by averaging the corresponding values for all lesions for each subject. The following parameters and features were extracted:

i. $\quad$ Shape Parameters: 1) X-coordinate maximum length, 2) Y-coordinate maximum length, 3) area, 4) perimeter, 5) perimeter2/area, 6) eccentricity, 7) equivalence diameter, 8) major axis length, 9) minor axis length, 10) centroid, 11) convex area, and 12) orientation. 
ii. Statistical Features ${ }^{[14,21]}$ : a) mean, b) variance, c) median value, d) skewness, e) kurtosis, f) energy and g) entropy.

iii. Spatial Gray Level Dependence Matrices (SGLDM) as proposed by Haralick et al. ${ }^{[14]}$ : a) angular second moment (ASM), b) contrast, c) correlation, d) sum of squares variance (SOSV), e) inverse difference moment (IDM), f) sum average (SA), g) sum variance (SV), h) sum entropy (SE), i) entropy, j) difference variance (DV), k) difference entropy (DE), and l) information measures of correlation (IMC). For a chosen distance $\mathrm{d}$ (in this work was used) and for angles $\theta=0^{\circ}, 45^{\circ}, 90^{\circ}$, and $135^{\circ}$, we computed four values for each of the above texture measures. Each feature was computed using a distance of one pixel. Then for each feature the mean values and the range of values were computed, and were used as two different feature sets.

iv. Gray Level Difference Statistics (GLDS ${ }^{[37]}$ : a) homogeneity, b) contrast, c) energy, d) entropy, and e) mean. The above features were calculated for displacements $\delta=(0,1),(1,1),(1,0)(1,-1)$, where $\delta \equiv(\Delta x, \Delta y)$, and their mean values were taken.

v. Neighborhood Gray Tone Difference Matrix (NGTDM) ${ }^{[1]}$ : a) coarseness, b) contrast, c) busyness, d) complexity, and e) strength.

vi. Statistical Feature Matrix (SFM) ${ }^{[38]}$ : a) coarseness, b) contrast, c) periodicity, and d) roughness.

vii. Laws Texture Energy Measures (LTEM) ${ }^{[38]}$ : LL-texture energy from LL kernel, EE-texture energy from EE-kernel, SS-texture energy from SS-kernel, LE-average texture energy from LE and EL kernels, ES-average texture energy from ES and SE kernels, and LS-average texture energy from LS and SL kernels.

viii. Fractal Dimension Texture Analysis (FDTA) ${ }^{[38]}$ : The Hurst coefficients for dimensions 4, 3 and 2 were computed.

ix. Fourier Power Spectrum (FPS) ${ }^{[38]}$ : a) radial sum, and b) angular sum.

\subsection{Evaluation metrics and statistical analysis}

Distance Measures: In order to identify the most discriminant features and cross-evaluate between the different normalization methods the following distance measures for each feature were computed:

1) A simple figure of merit for the discriminatory value of each individual feature could be defined by computing the distance between two classes for each feature as follows ${ }^{[6]}$ :

$$
D_{\text {FEAT }}=\sum_{i=0}^{N} \frac{\left|m_{g_{i}}-m_{f_{i}}\right|}{\sqrt{\sigma_{g i}^{2}-\sigma_{f i}^{2}}}
$$

where $\mathrm{N}$ is the number of features, and $m_{g_{i}}$ and $m_{f_{i}}$ represent the mean feature values, and $\sigma_{g_{i}}$ and $\sigma_{f_{i}}$ represent the standard deviation feature values of the original and normalized features respectively.

2) Furthermore, a variation of the Hausdorff distance, $D_{H D}$, between two sets (original, $g(x, y)$, and normalized $f(x, y)$, images) was calculated ${ }^{[4]}$. It reflects the maximum mismatch between the original and the normalized images.

$$
D_{H D}=M A X|g(x, y)-f(x, y)|
$$


3) The Mahalanobis distance ${ }^{[23]}$ was calculated between the original and the normalized images. It is based on correlations between variables by which different patterns can be identified and analyzed. The Mahalanobis distance, $D_{M}$, can be defined as a dissimilarity measure between two random vectors (original $(g)$, and normalized $(f)$ ) of the same distribution with the covariance matrix $\mathrm{S}$ as:

$$
D_{M}=\sqrt{(\vec{g}-\vec{f})^{T} S^{-1}(\vec{g}-\vec{f})}
$$

4) Kullback-Leibler Divergence: Instead of measuring the similarity, we measure the dissimilarity of two histograms and by the Kullback-Leibler Divergence $\left(\mathrm{D}_{\mathrm{KLD}}\right)$ or relative entropy between two distributions ${ }^{[29]}$, given by:

$$
D_{K L D}=\sum_{i} P_{g}(i) \log \frac{P_{g}(i)}{P_{f}(i)}
$$

where, $P_{g}$ and $P_{f}$ are the probability distributions corresponding to $g(x, y)$ and $f(x, y)$, respectively, and represents the gray level index. The $\mathrm{D}_{\mathrm{KLD}}$ yields a positive value. A higher score indicates a higher dissimilarity, hence a higher possibility of a histogram or a feature shift ${ }^{[29]}$.

5) The structural similarity index (SSIN) between two images ${ }^{[36]}$, which models any distortion as a combination of three different factors, which are: loss of correlation, luminance distortion, and contrast distortion and is derived in generalized form as:

$$
\operatorname{SSIN}=\frac{\left(2 m_{g} m_{f}+c_{1}\right)\left(2 \sigma_{g f}+c_{2}\right)}{\left(m_{g}{ }^{2}+m_{f}{ }^{2}+c_{1}\right)\left(\sigma_{g}^{2}+\sigma_{f}^{2}+c_{2}\right)}-1<\operatorname{SSIN}<1
$$

where $m_{g}$, and $m_{f}$ represent the mean of the original and normalized image values, with their standard deviations, $\sigma_{g}$, and $\sigma_{f}$, of the original and normalized values of the analysis window, and $\sigma_{g f}$, represents the covariance between the original and transformed images. The constants $c_{1}=0.01 d r$, and $c_{2}=0.03 d r$, with $d r=255$, represents the dynamic rage of the images. The range of values for the SSIN lies between -1 , for a bad and 1 for a good similarity between the original and normalized images respectively. It is computed for a sliding window of size $8 \times 8$ without overlapping.

The non-parametric Wilcoxon rank sum test ${ }^{[1]}$ was used in order to detect if for each texture feature a significant difference (S) or not (NS) exists between the original and the normalized images at $p<0.05$. The test was implemented using the MATLAB ${ }^{\circledR}$ ranksum(x, y) which performs a two-sided rank sum test of the null hypothesis that data in the vectors $\mathrm{x}$ and $\mathrm{y}$ are independent samples from identical continuous distributions with equal medians, against the alternative that they do not have equal medians. We also calculated the coefficient of variation, $C V \%$, which describes the difference as a percentage of the pooled mean value with $C V \%=(\sigma / \mu) \times 100$, where $\sigma$, and $\mu$, represent the standard deviation and the mean values of the sample ${ }^{[26]}$. Furthermore, the correlation coefficient, $\rho$, between the original and the normalized features was calculated, which returns the normalized covariance matrix.

\section{Results}

In Fig. 1 we present an example of MRI pre-processing steps of a 34 year old female patient at 0 (EDSS=1) and 6-12 $($ EDSS=2) months in the left (see Fig.1(a)-(e)) and right (see Fig. 1(f)-(l)) columns respectively. We present the first slide 
from the MRI image sequences where the manually selected areas for the BWM and GWM are shown in Fig. 1(a) and Fig. 1(f) for the 0 and 6-12 months sequences respectively. These values are used to perform the normalization process in all the consecutive brain slices as described in section 2.3 for the HN method. Figure 1(b) and 1(c) present the MRI image of the same patient at 0 months, with one manually segmented lesion before normalization, and after normalization with the HN method respectively (and similarly in Fig. 1(g) and 1(h) for the same patient at 6-12 months). In Fig. 1(d) and 1(i) we illustrate the extracted lesion at 0 months (median (IQR): 92 (11)), and the three extracted lesions at 6-12 months (median (IQR): 123 (13), 96 (11), 98 (11)) resized by a factor of 3, respectively. The corresponding normalized lesion histogram at 0 months (median (IQR): 86(52)), is given in Fig. 1(e), and the corresponding normalized histograms for the three lesions at 6-12 months (median (IQR): 98 (55), 83 (31), 78 (22)), are given in Fig. 1 (j)-(1) respectively.

Figure 2(a) presents an MRI brain image of the same patient as in Fig. 1 at 0 months with a different lesion before normalization resized by a factor of 4 as shown in Fig. 2(b). The original lesion histogram is presented in Fig. 2(c) whereas Fig. 2(d) through Fig. 2(i) present the different lesion histograms generated after the application of each normalization method for the cases of CSN, IS, HS, HN, GKN and HE, respectively.

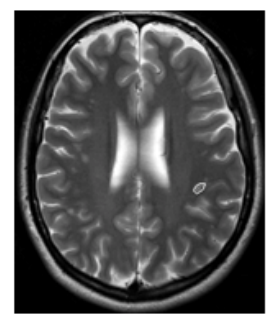

(a) MRI image at 0 months

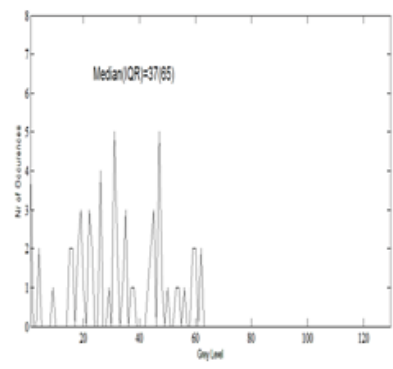

(d) CSN normalised histogram

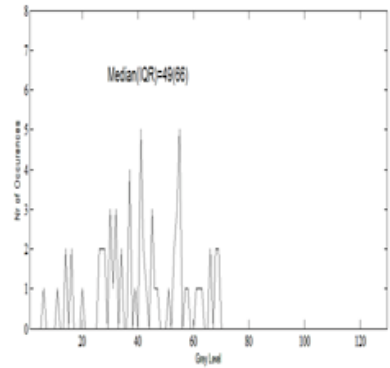

(g) HN normalised histogram

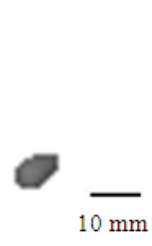

(b) Extracted lesion
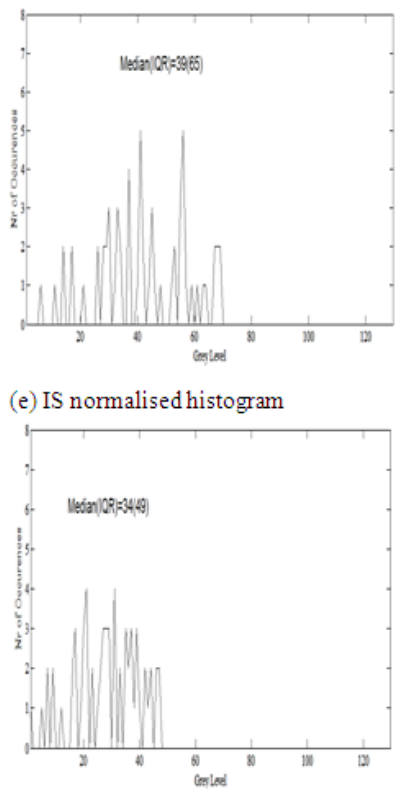

(h) GKN normalised histogram

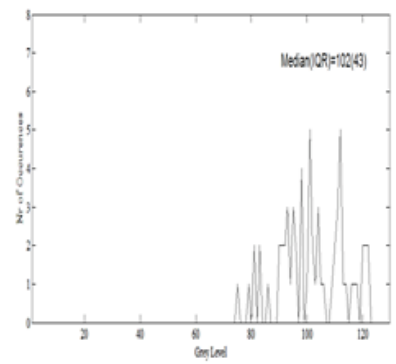

(c) Original lesion histogram
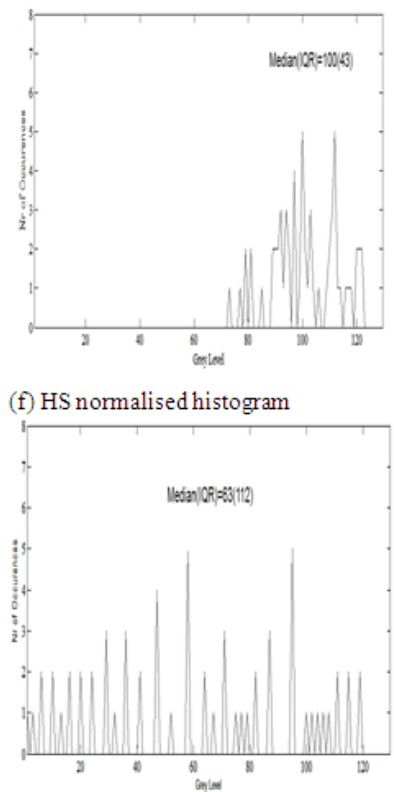

(i) HE normalised histogram

Figure 2. An example of interscan intensity MRI imaging normalization methods. (a) MR brain image of the same patient as in Fig. 1 at 0 months with a different lesion (BWM=6, GWM=245), (b) the extracted lesion before normalization resized by a factor of 4, (c) lesion histogram before normalization (median (IQR): 102 (43)), (d) normalized lesion histogram with the CSN method (median (IQR): 37 (65)), (e) normalized lesion histogram with the IS method (median (IQR): 39 (65)), (f) normalized lesion histogram with the HS method (median (IQR): 100 (43)), (g) normalized lesion histogram with the HN method (IQR): 49 (66)), (h) normalized lesion histogram with the GKN method (IQR): 34 (49)), and (i) normalized lesion histogram with the HE method (IQR): 63 (112)). 
Table 1 presents the median values of texture features at 0 versus 6-12 months extracted from the original (Orig.) and the normalized MRI images (CSN, IS, HS, HN, GKN, HE). The Wilcoxon rank sum test was performed on the texture features of the original and the normalized images for 0 versus 6-12 months. It is shown in Table 1, that the different features were affected differently by the normalization process. For example, for the features median and contrast there was significant difference between 0 and 6-12 months for the original images but not for the HN normalized. On the other hand for the feature complexity there was no significant difference between 0 and 6-12 months for the original image, but there was for the HN normalized images.

Table 1. Median Values of Texture Features and Statistical Analysis at 0 versus 6-12 Months (-/-) for All Lesions for the Different Normalization Methods Investigated (Number of Subjects N=38)

\begin{tabular}{|c|c|c|c|c|c|c|c|c|c|c|}
\hline Method & Median & S/NS & STD & S/NS & Contr & S/NS & Entr & S/NS & DE & S/NS \\
\hline Orig. & $127 / 117$ & $\mathrm{~S}(0.006)$ & $16 / 15$ & $\mathrm{NS}(0.10)$ & $91 / 74$ & $\mathrm{~S}(0.04)$ & $5.1 / 5.1$ & $\mathrm{NS}(0.9)$ & $2.7 / 2.6$ & $\mathrm{NS}(0.7)$ \\
\hline $\mathrm{CSN}$ & $119 / 107$ & $\mathrm{~S}(0.03)$ & $22 / 21$ & $\mathrm{NS}(0.22)$ & $124 / 94$ & $\mathrm{NS}(0.15)$ & $5.7 / 5.7$ & $\mathrm{NS}(0.8)$ & $2.6 / 2.5$ & $\mathrm{NS}(0.3)$ \\
\hline IS & $114 / 108$ & $S(0.03)$ & $19 / 18$ & $\mathrm{NS}(0.21)$ & $87 / 77$ & $\mathrm{NS}(0.22)$ & $5.7 / 5.6$ & $\mathrm{NS}(0.8)$ & $2.8 / 2.6$ & $\mathrm{~S}(0.04)$ \\
\hline HS & $115 / 109$ & $\mathrm{~S}(0.04)$ & $19 / 17$ & $\mathrm{NS}(0.24)$ & $112 / 87$ & $\mathrm{NS}(0.31)$ & $5.7 / 5.7$ & $\mathrm{NS}(0.8)$ & $2.4 / 2.7$ & $\mathrm{~S}(0.03)$ \\
\hline $\mathrm{HN}$ & $115 / 106$ & $\mathrm{NS}(0.06)$ & $20 / 19$ & $\mathrm{NS}(0.47)$ & $116 / 112$ & $\mathrm{NS}(0.3)$ & $5.7 / 5.7$ & $\mathrm{NS}(0.8)$ & $2.7 / 2.9$ & $S(0.02)$ \\
\hline GKN & $117 / 114$ & $\mathrm{NS}(0.2)$ & $19 / 18$ & $\mathrm{NS}(0.36)$ & $119 / 114$ & $\mathrm{NS}(0.62)$ & $5.7 / 5.7$ & $\mathrm{NS}(0.7)$ & $2.5 / 2.7$ & $S(0.05)$ \\
\hline $\mathrm{HE}$ & $119 / 116$ & $\mathrm{~S}(0.003)$ & $10 / 9$ & $\mathrm{NS}(0.08)$ & $55 / 54$ & $\mathrm{NS}(0.14)$ & $5.3 / 5.4$ & $\mathrm{NS}(0.3)$ & $2.3 / 2.4$ & $\mathrm{NS}(0.3)$ \\
\hline Method & PERD & S/NS & SV & S/NS & BUS & S/NS & СОМР & S/NS & IDM & S/NS \\
\hline Orig. & $0.63 / 0.63$ & $\mathrm{NS}(0.3)$ & $172 / 147$ & $\mathrm{~S}(0.03)$ & $11 / 13$ & $\mathrm{NS}(0.2)$ & $12 / 23$ & $\mathrm{NS}(0.33)$ & $0.145 / 154$ & $\mathrm{~S}(0.03)$ \\
\hline $\mathrm{CSN}$ & $0.66 / 0.67$ & $\mathrm{NS}(0.1)$ & $159 / 153$ & $\mathrm{NS}(0.07)$ & $10 / 19$ & $\mathrm{NS}(0.31)$ & $11 / 17$ & $\mathrm{NS}(0.12)$ & $0.161 / 1.72$ & NS (0.22) \\
\hline IS & $0.64 / 0.65$ & $\mathrm{~S}(0.04)$ & $186 / 177$ & $\mathrm{~S}(0.03)$ & $12 / 16$ & $\mathrm{~S}(0.02)$ & $13 / 19$ & $\mathrm{~S}(0.02)$ & $0.15 / 0.16$ & $S(0.04)$ \\
\hline HS & $0.65 / 0.67$ & $\mathrm{NS}(0.19)$ & $217 / 204$ & $\mathrm{NS}(0.24)$ & $15 / 17$ & $\mathrm{NS}(0.7)$ & $21 / 24$ & $\mathrm{NS}(0.3)$ & $0.14 / 0.15$ & $\mathrm{NS}(0.07)$ \\
\hline $\mathrm{HN}$ & $0.64 / 0.65$ & $\mathrm{NS}(0.02)$ & $154 / 157$ & $\mathrm{~S}(0.03)$ & $14 / 17$ & $\mathrm{NS}(0.02)$ & $13 / 22$ & $S(0.04)$ & $0.24 / 0.25$ & $\mathrm{~S}(0.03)$ \\
\hline GKN & $0.65 / 0.66$ & $\mathrm{NS}(0.1)$ & $123 / 118$ & $\mathrm{NS}(0.2)$ & $15 / 16$ & $\mathrm{NS}(0.3)$ & $15 / 17$ & $\mathrm{NS}(0.3)$ & $0.16 / 0.17$ & $\mathrm{NS}(0.09)$ \\
\hline $\mathrm{HE}$ & $0.66 / 0.67$ & $\mathrm{NS}(0.1)$ & $166 / 172$ & $\mathrm{~S}(0.003)$ & $13 / 14$ & $\mathrm{NS}(0.56)$ & $18 / 21$ & $\mathrm{NS}(0.09)$ & $0.14 / 0.15$ & $\mathrm{NS}(0.31)$ \\
\hline
\end{tabular}

Note. CSN: Contrast Stretch Normalization, IS: Intensity Scaling, HS: Histogram Stretching, HN: Histogram Normalization, GKN: Gaussian Kernel Normalization, HE: Histogram Equalization. STD: Standard Deviation, Contr.: Contrast, Entr.: Entropy, DE: Difference entropy, PERD: Periodicity, SV: Sum variance, BUS: Busyness, COMP: Complexity, IDM: Inverse Difference Moment, S/NS: Significantly (S) different at $p<=0.05$, and non-significantly different (NS) at $p>0.05$, with the $p$ value given in parentheses.

Table 2 shows the results of the Wilcoxon rank sum test performed on the texture features extracted from all lesions between the original versus the normalized images for both 0 and 6-12 months. The test was performed to check if significant differences exist between the features computed on the original versus the normalized lesions. Normalization methods that resulted with the most significant number of features after normalization were the $\mathrm{HN}$ for 0 months and the HS, HN and GKN for 6-12 months. The rest of the normalization methods gave a lower number of significantly different features. Features that showed a significant difference after normalization were the median, entropy (Entr.), and complexity (COMP).

Table 3 shows the median (IQR) values for different distance measures for the six different normalization methods investigated for the lesion ROIS at 0/6-12 months for all subjects investigated $(\mathrm{N}=38)$. It is shown that the $\mathrm{HN}$ image normalization method exhibits small distances ( $D_{\text {FEAT }}=0.13(0.19) / 0.11(0.16), D_{H D}=0.17(0.21) / 0.15(0.17)$, 
$\left.D_{M}=0.19(0.16) / 0.21(0.17), D_{K L D}=0.053(0.08) / 0.062(0.14)\right)$, a large $\operatorname{SSIN}(\operatorname{SSIN}=0.98(0.08)) / 0.97(0.11)$, the smallest coefficient of variation $(C V \%=14.4 / 17.7$, for 0 and 6-12 months respectively) and the largest correlation coefficient ( $\rho=0.91 / 0.89$ ), for 0/6-12 months, when compared with the rest of the normalization methods.

Table 2. Wilcoxon Rank Sum Test Performed on the Texture Features Extracted from All Lesions between The Original and The Normalized Images At 0 And 6-12 Months

\begin{tabular}{|c|c|c|c|c|c|c|c|c|c|c|}
\hline Method & Median & STD & Contr & Entr & DE & IDM & PERD & SV & BUS & COMP \\
\hline & \multicolumn{10}{|c|}{ Original vs Normalized at 0 months } \\
\hline CSN & $\mathrm{S}$ & $\mathrm{S}$ & $\mathrm{S}$ & $\mathrm{S}$ & $\mathrm{S}$ & $\mathrm{S}$ & NS & NS & $\mathrm{S}$ & S \\
\hline IS & S & S & $\mathrm{S}$ & $\mathrm{S}$ & S & NS & $\mathrm{S}$ & NS & $\mathrm{S}$ & S \\
\hline HS & S & S & S & $\mathrm{S}$ & S & $\mathrm{S}$ & NS & NS & $\mathrm{S}$ & S \\
\hline $\mathrm{HN}$ & S & S & S & $\mathrm{S}$ & S & $\mathrm{S}$ & S & $\mathrm{S}$ & S & S \\
\hline GKN & $\mathrm{S}$ & S & NS & S & S & S & S & S & NS & S \\
\hline \multirow[t]{2}{*}{$\mathrm{HE}$} & $\mathrm{S}$ & NS & $\mathrm{S}$ & $\mathrm{S}$ & NS & $\mathrm{S}$ & NS & NS & $\mathrm{S}$ & S \\
\hline & \multicolumn{10}{|c|}{ Original vs Normalized at 6-12 months } \\
\hline CSN & $\mathrm{S}$ & $\mathrm{S}$ & $\mathrm{S}$ & $\mathrm{S}$ & $\mathrm{S}$ & $\mathrm{S}$ & NS & NS & $\mathrm{S}$ & $\mathrm{S}$ \\
\hline IS & S & S & NS & $\mathrm{S}$ & S & S & $\mathrm{S}$ & NS & NS & S \\
\hline HS & $\mathrm{S}$ & $\mathrm{S}$ & $\mathrm{S}$ & $\mathrm{S}$ & $\mathrm{S}$ & $\mathrm{S}$ & $\mathrm{S}$ & NS & S & S \\
\hline $\mathrm{HN}$ & $\mathrm{S}$ & $\mathrm{S}$ & NS & $\mathrm{S}$ & $\mathrm{S}$ & $\mathrm{S}$ & S & $\mathrm{S}$ & $\mathrm{S}$ & $\mathrm{S}$ \\
\hline GKN & NS & $\mathrm{S}$ & $\mathrm{S}$ & $\mathrm{S}$ & $\mathrm{S}$ & $\mathrm{S}$ & $\mathrm{S}$ & $\mathrm{S}$ & NS & $\mathrm{S}$ \\
\hline HE & $\mathrm{S}$ & NS & $S$ & $\mathrm{~S}$ & NS & $\mathrm{S}$ & NS & NS & $\mathrm{S}$ & $\mathrm{S}$ \\
\hline
\end{tabular}

Table 3. Distance Measures For The Six Different Normalization Methods Investigated For The Lesion ROIS AT 0/6-12 Months (median (IQR)) (Number of Subjects N=38)

\begin{tabular}{llllllll}
\hline $\begin{array}{l}\text { Method vs } \\
\text { Normalized }\end{array}$ & $\mathbf{D}_{\text {FEAT }}$ & $\mathbf{D}_{\text {HD }}$ & $\mathbf{D}_{\mathbf{M}}$ & $\mathbf{D}_{\text {KLD }}$ & SSIN & CV\% & $\rho$ \\
\hline \multirow{2}{*}{ CSN } & $0.19(0.61) /$ & $0.28(0.26) /$ & $0.21(0.19) /$ & $0.21(0.06) /$ & $0.87(0.26) /$ & $18.2 /$ & $0.37 /$ \\
& $0.21(0.17)$ & $0.31(0.26)$ & $0.23(0.22)$ & $0.23(0.17)$ & $0.88(0.27)$ & 19.1 & 0.46 \\
\multirow{2}{*}{$\mathrm{IS}$} & $0.43(0.42) /$ & $0.32(0.29) /$ & $0.40(0.31) /$ & $0.06(0.16) /$ & $0.96(0.17) /$ & $18.1 /$ & $0.38 /$ \\
& $0.41(0.39)$ & $0.29(0.25)$ & $0.39(0.27)$ & $0.08(0.15)$ & $0.96(0.26)$ & 16.9 & 0.45 \\
$\mathrm{HS}$ & $0.32(0.37) /$ & $0.35(0.33) /$ & $0.33(0.29) /$ & $0.22(0.07) /$ & $0.92(0.12) /$ & $17.67 /$ & $0.41 /$ \\
& $0.28(0.33)$ & $0.38(0.31)$ & $0.34(0.26)$ & $0.21(0.12)$ & $0.92(0.19)$ & 18.7 & 0.49 \\
$\mathrm{HN}$ & $0.13(0.19) /$ & $0.17(0.21) /$ & $0.19(0.16) /$ & $0.053(0.08) /$ & $0.98(0.08) /$ & $14.4 /$ & $0.91 /$ \\
& $0.11(0.16)$ & $0.15(0.17)$ & $0.21(0.17)$ & $0.062(0.14)$ & $0.97(0.11)$ & 17.7 & 0.89 \\
$\mathrm{GKN}$ & $0.35(0.46) /$ & $0.37(0.27) /$ & $0.32(0.44) /$ & $0.22(0.07) /$ & $0.92(0.17) /$ & $17.2 /$ & $0.69 /$ \\
& $0.34(0.39)$ & $0.32(0.23)$ & $0.28(0.39)$ & $0.24(0.13)$ & $0.90(0.21)$ & 19.1 & 0.66 \\
$\mathrm{HE}$ & $0.17(0.39)$ & $0.19(0.22) /$ & $0.20(0.29) /$ & $0.32(0.47) /$ & $0.88(0.31) /$ & $6.61 /$ & $0.88 /$ \\
& $0.21(0.37)$ & $0.21(0.24)$ & $0.22(0.28)$ & $0.39(0.47)$ & $0.87(0.33)$ & 7.12 & 0.81 \\
\hline
\end{tabular}

Note. CSN: Contrast stretch normalization, IS: Intensity scaling, HS: Histogram stretching, HN: Histogram normalization, GKN: Gaussian kernel normalization, HE: Histogram equalization. $D_{F E A T}$ Feature distance, $D_{H D}$ : Hausdorff distance, $D_{M}$ : Mahalanobis distance, $D_{K L D}:$ Kullback-Leibler Divergence distance, SSIN: Structural similarity index, $C V \%$ : Coefficient of Variation, $\rho$ : Correlation coefficient

Figure 3 presents box plots for the $D_{K L D}$ for 0 and 6-12 months for all the normalization methods investigated. It is clearly illustrated that the HN method exhibits the smallest $D_{K L D}$ at both 0 and 6-12 months (median (IQR) values of 0.053(0.08) 
and $0.062(0.14)$ respectively) which means that this method brings about the smallest divergence to the original image histogram.

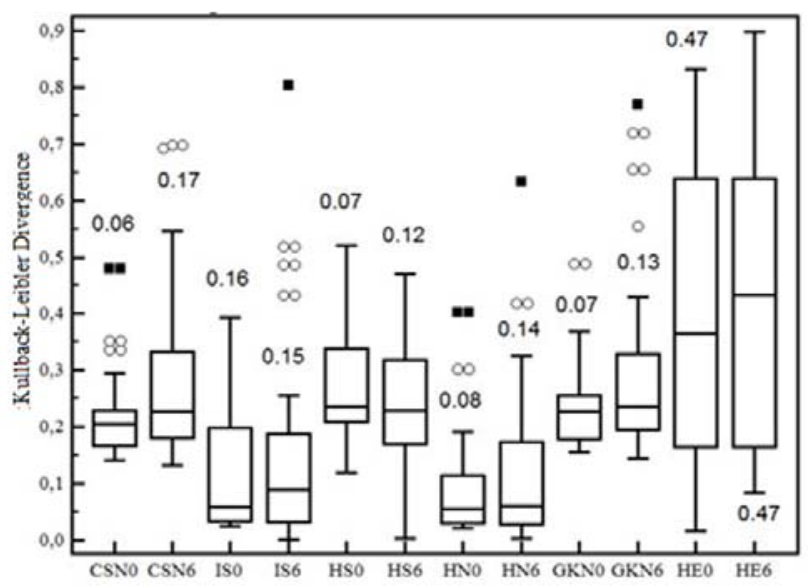

Figure 3. Box plots for the Kullback-Leibler Divergence $\left(\mathrm{D}_{\mathrm{KLD}}\right)$ for the 6 different MRI image normalization methods for 0 and 6-12 months for the $38 \mathrm{MS}$ subjects studied. IQR values are shown above the box plots. In each plot we display the median, lower, and upper quartiles and confidence interval around the median. Straight lines connect the nearest observations with 1.5 of the Inter-Quartile Range (IQR) of the lower and upper quartiles. Unfilled rectangles indicate possible outliers with values beyond the ends of the $1.5 \mathrm{x}$ IQR.

\section{Discusion}

The objective of this study was to implement and investigate six different, previously proposed image normalization methods and propose the most suitable for the analysis of brain MRI images in the case of MS. The image normalization methods investigated were the following: Contrast Stretch Normalization (CSN), Intensity Scaling (IS), Histogram Stretching (HS), Histogram Normalization (HN), Gaussian Kernel Normalization (GKN) and Histogram Equalization (HE). Data presented in Tables 1 and 2I suggest that the normalization procedure applied on brain MR images significantly affects quantitative texture analysis and that different normalization techniques have variant effects on discrete texture features. Various different features were considered in the current study, irrespective of their relative ability with regard to MS lesion load assessment, to allow discrimination of the technique that better alleviates the problems imposed by intra- and inter-scan signal intensity variations. The main findings of this study can be summarized as follows:

1) Lesion texture features were affected differently by the normalization process for both the 0 and 6-12 months MRI scans (see also Table 1). For example, for the features median and contrast there was significant difference between 0 and 6-12 months for the original images but not for the HN normalized. On the other hand for the feature complexity there was no significant difference between 0 and 6-12 months for the original image, but there was for the $\mathrm{HN}$ normalized images.

2) The statistical lesion feature analysis between the original and the normalized images showed that the HN method gave the highest number of significant features after normalization for both the 0 and 6-12 months MRI scans (see also Table 2).

3) The HN normalization method gave the best performance compared to the other normalization methods with respect to the distance measures, structural similarity index, coefficient of variation, and correlation coefficient between the original and normalized 0 and 6-12 months MRI scans (see also Table 3). 
Thus, based on the above findings it is recommended that the simple HN normalization method could be used for assessing the MS lesion load in the studied patient group. This normalization method allows for scanner instrumentation variations to be partly compensated and thereby facilitates meaningful comparisons between MRI data sets obtained at different times and from different subjects. The method does not depend on knowledge of the applied scanner calibration procedure and thus can be used on retrospectively collected data.

The proposed HN normalization method was used in a recent study carried out by our group. The use of multi-scale Amplitude Modulation-Frequency Modulation (AM-FM) texture analysis of MS using MRI images was investigated on the same image set of MRI scans presented in this paper ${ }^{[20]}$. The instantaneous amplitude (IA), the magnitude of the instantaneous frequency (IF) and the IF angle were extracted from each segmented region at different scales. The findings suggest that AM-FM characteristics succeed in differentiating (i) between NWM and lesions, (ii) between NAWM and lesions, and (iii) between NWM and NAWM. A support vector machines (SVM) classifier succeeded in differentiating between patients that, two years after the initial MRI scan, acquired an EDSS $<=2$ from those with EDSS $>2$ (correct classification rate $=86 \%$ ). The best classification results were obtained from including the combination of the low-scale IA \& IF magnitude with the medium-scale IA. The AM-FM features provide complementary information to classical texture analysis features like the gray scale median (GSM), contrast, and coarseness.

Several other studies with more complicated algorithms and very promising results have been proposed in the literature for MRI normalization that are briefly discussed below.

The influence of MRI acquisition protocols, and gray level normalization methods on texture classification ${ }^{[7]}$ were evaluated. Image grey levels were normalized to limit image intensities to between $[m-3 \sigma, m+3 \sigma]$ for each ROI, where $m$ is the mean grey level, and $\sigma$ is the standard deviation. This normalization scheme reported the best classification results in the case of MR imaging of soft cheeses and having no relationship between classification errors and MR acquisition protocols. The results also showed that the normalization method and the acquisition protocol influence the effectiveness of the classification. More specifically, if no normalization was applied, the classification errors depend on the MR acquisition protocols. However, when using normalization, no relationship was observed and the classification results were significantly improved.

A histogram matching method was proposed ${ }^{[35]}$ for correcting the variations in scanner sensitivity due to differences in scanner performance. It was shown that the method reduced the variation in white matter intensities from $7.5 \%$ to $2.5 \%$ and provided a method to remove the threshold dependency in lesion volume measurement with global thresholding in patients with MS.

Furthermore, the utility of even order derivative analysis in MRI histogram normalization has been demonstrated ${ }^{[5]}$. It was shown that good white matter peak discrimination was achieved even when significant overlap existed between gray matter and white matter peaks, as this is the case with the T2-weighted brain images. Furthermore, the ability of the normalization procedure to correct the global intensity variations over time was demonstrated by the high degree of reproducibility of an automatic brain segmentation algorithm following intensity normalization.

In another study ${ }^{[25]}$, an image post processing method for integrating multiple serial MRI scans into a volume for direct quantitative evaluation of the temporal intensity profiles was proposed. A significant error reduction was observed when applying tissue specific inter-scan intensity normalization, suggesting that intensity variations above $3 \%$ can be reliably detected.

Nyul et al. ${ }^{[28]}$ proposed a method consisting of a training stage to find the parameters of the standard scale and a transformation stage that maps the histograms of candidate volumes to the standard histogram scale in a piece-wise linear manner. The scale has been shown to be relatively stable across different variations of the piece-wise intervals. Later, the 
same authors proposed solutions for the limitations in their earlier study ${ }^{[27]}$. The effectiveness of the above method proposed ${ }^{[28]}$, was later evaluated ${ }^{[32]}$ for rendering. It was demonstrated that lesion segmentation is more accurate after the application of the normalization method.

\section{Concluding remarks}

MRI analysis has become a powerful tool in the diagnosis of brain disease ${ }^{[11,39]}$. Pixel intensity variations between the same and consecutive MRI scans i.e. intra-scan and inter-scan variations, complicate the method of quantitative MRI analysis ${ }^{[25]}$. Improvements in the measurement and pre-processing of the image may therefore have a significant impact in the clinical diagnosis, image analysis, and computer aided diagnosis. In the current case study of $38 \mathrm{MS}$ patients, it was shown that the simple HN method of histogram intensity normalization proposed ${ }^{[22]}$ can help in this direction. This will enable a more accurate computation of texture features that may provide information for the earlier differentiation between normal tissue and MS lesions and in assessing disease progression. MRI texture analyses of lesions in MS can also help in specifying the types of MS (CIS, primary progressive, progressive-relapsing, relapsing-remitting and secondary progressive based on the differences in analyses between the groups. Therefore, the proposed normalization method may be useful in the inter-comparison of both serial and cross-sectional MRI data sets from MS patients.-.Further research work on a larger number of subjects is required for validating the results of this study and for assessing additional algorithms which may be found suitable for normalizing brain MRI scans of MS patients.

\section{Acknowledgment}

This work was funded through the project Quantitative and Qualitative Analysis of MRI Brain Images TПЕ/OPIZO/ 0308(BIE)/15, 12/2008-12/2010, of the Program for Research and Technological Development 2007-2013, of the Research Promotion Foundation of Cyprus.

\section{References}

[1] Altman DG. Practical Statistics for Medical Research. 1991; London, U.K.: Chapman and Hall.

[2] Amadasun M, King R. Textural features corresponding to textural properties. IEEE Trans. Syst., Man. Cybern. 1989; 19(5): 1264-1274. http://dx.doi.org/10.1109/21.44046

[3] Bakshi R, Thompson AJ, Rocca MA, Pelletier D, Dousset V, Barkhof F, et al. MRI in multiple sclerosis: current status and future prospects. Lancet Neurol. 2008; 7: 615-625. http://dx.doi.org/10.1016/S1474-4422(08)70137-6

[4] Chalana V, Kim Y. A methodology for evaluation of boundary detection algorithms on medical images. IEEE Trans. Med. Imag. 1997; 16(5): 642-652. PMid:9368120 http://dx.doi.org/10.1109/42.640755

[5] Christensen J.D. Normalization of brain magnetic resonance images using histogram even-order derivative analysis. Mag. Reson. Imag. 2003; 21: 817-820. http://dx.doi.org/10.1016/S0730-725X(03)00102-4

[6] Christodoulou C, Pattichis C, Pantziaris M, Nicolaides A. Texture-Based Classification of Atherosclerotic Carotid Plaques. IEEE Trans. Med. Imag. 2003; 22(7): 902-912. PMid:12906244 http://dx.doi.org/10.1109/TMI.2003.815066

[7] Collewet G Strzelecki M and Marriette F. Influence of MRI acquisition protocols and image intensity normalization methods on texture classification. Magn. Reson. Imag. 2004; 22: 81-91. PMid:14972397 http://dx.doi.org/10.1016/j.mri.2003.09.001

[8] Conover WJ. Practical nonparametric statistics. 1999; 3rd Ed., New York: John Wiley \& Sons.

[9] DeCarli C, Murphy DGM, Teichberg D, Campbell G, Sobering GS. Local histogram correction of MRI spatially dependent image pixel intensity nonuniformity. J. Magn. Res. Imag. 1996; 6(3): 519-528. http://dx.doi.org/10.1002/jmri.1880060316

[10] Dehmeshki J, Barker GJ, Tofts PS. Classifications of disease subgroups and correlation with disease severity using magnetic resonance imaging whole-brain histograms: Application to magnetization transfer ratios and multiple sclerosis. IEEE Trans. Med. Imag. 2002; 21(4): 320-331. PMid:12022620 http://dx.doi.org/10.1109/TMI.2002.1000256

[11] Fazekas F, Barkof F, Filippi M, Grossman RI, Li DKB., McDonald WI, McFarland HF, Patty DW, Simon JH, Wolinsky JS, Miller $\mathrm{DH}$. The contribution of magnetic resonance imaging to the diagnosis of multiple sclerosis. Neur. 1999; 53: 448-456. 
[12] Filippi M, Paty DW, Kappos L, Barkhof F, Compston DA, Thompson AJ, Zhao GJ, Wiles CM, McDonald WI, Miller DH. Correlations between changes in disability and T2-weighted brain MRI activity in multiple sclerosis: a follow-up study. Neur. 1995; 45: 255-260.

[13] Gonzalez RC. Woods R.E. Digital Image processing. 2002;2nd Ed. Prentice Hall.

[14] Haralick R.M, Shanmugam K, Dinstein I. Texture features for image classification. IEEE Trans. Syst. Man. and Cybernetics. 1973; SMC-3: 610-621. http://dx.doi.org/10.1109/TSMC.1973.4309314

[15] Harrison LCV, Raunio M, Holli KK, Luukkaala T, Savio S, Elovaara I, Soimakallio S, Eskola HJ, Dastidar P. MRI Texture analysis in multiple sclerosis: Toward a clinical analysis protocol. Acad. Radiol. 2010; 17: 696-707. PMid:20457414 http://dx.doi.org/10.1016/j.acra.2010.01.005

[16] Herlidou-Meme S, Constans JM, Carsin B, Olivie D, Eliat PA et al. MRI texture analysis on texture test objects, normal brain and intracranial tumors. Mag. Res. Imag. 2003; 21: 989-993. http://dx.doi.org/10.1016/S0730-725X(03)00212-1

[17] Heush G, Gardinaux F. Lighting normalisation algorithms for face verification. IDIAP research institute. Internal report. 2005; IDIAP-com-03:1-40.

[18] Koptenko S. Contrast Stretch and Normalization. 2006; Matlab FEX. http://www.mathworks.com/matlabcentral/fileexchange/11429.

[19] Loizou CP, Kyriacou EC, Seimenis I, Pantziaris M, Christodoulou C, Pattichis MS, Pattichis CS. Brain white matter lesions classification in multiple sclerosis subjects for the prognosis of future disability. 12th Eng. Applications of Neural Networks (EANN)/7th Artificial Intelligence Applications and Innovations (AIAI). 2001; 400-409; Corfu, Greece, Sept. 15-18, Part II, IFIP AICT 364.

[20] Loizou CP, Murray V, Pattichis MS, Seimenis I, Pantziaris M, Pattichis CS. Multi-scale Amplitude Modulation-Frequency Modulation (AM-FM) texture analysis of multiple sclerosis in brain MRI images. IEEE Trans. Inform. Tech. Biomed. 2011; 15(1): 119-129. PMid:21062681 http://dx.doi.org/10.1109/TITB.2010.2091279

[21] Loizou CP, Pattichis CS, Seimenis I, Pantziaris M. Quantitative analysis of brain white matter lesions in multiple sclerosis subjects. 9th Int. Conf. Inform. Techn. Applic. Biomed.ITAB. 2009; 1-4; Larnaca, Cyprus. Nov. 5-7, 6 pages.

[22] Loizou CP, Patziaris M, Seimenis I, Pattichis CS. MRI intensity normalization in brain multiple sclerosis subjects. 9th Int. Conf. Inform. Techn. Applic. Biomed.-ITAB 2009;1-5;Larnaca, Cyprus. Nov. 5-7.

[23] Mahalanobis PC. On the generalized distance in statistics. Proc. of the National Institute of Sciences of India. 1936; $2(1): 49-55$. Available from: http://www.insa.ac.in/insa_pdf/20005b8c_49.pdf.

[24] McDonald WI, Compston A, Edan G, Goodkin D, Hartung HP, Lublin FD, et al. Recommended diagnostic criteria for multiple sclerosis: guidelines from the international panel on the diagnosis of multiple sclerosis. Ann. Neurol. 2001; 50: 121-127. PMid:11456302

[25] Meier DS Guttman CRG. Time-series analysis of MRI intensity patterns in multiple sclerosis. NeuroImag. 2003; 20: 1193-1209. http://dx.doi.org/10.1016/S1053-8119(03)00354-9

[26] Nixon M, Aguado A. Feature extraction \& image processing. 2000;Newnes.

[27] Nyul LG, Udupa JK, Zhang X, New variants of a method of MRI scale standardization. IEEE Trans. Med. Imag. 2000; 19(2): 143-150. PMid:10784285 http://dx.doi.org/10.1109/42.836373

[28] Nyul LG, Udupa JK. On standardizing the MR image intensity scale. Magn. Reson. Medic. 1999; 42 : $1072-1081$. http://dx.doi.org/10.1002/(SICI)1522-2594(199912)42:6<1072::AID-MRM11>3.0.CO;2-M

[29] Paranjape RB, Fundamental enhancement techniques. Handbook of Med. Imag. Proces. and Analysis. 2000;3-18. Academic Press. Ed. By Backman IN.

[30] Pluim JPW, Maintz JBA, Viergever MA. f-information measures in medical image registration. IEEE Trans. Med. Imag. 2004; 23(12): 1508-1516. PMid:15575408 http://dx.doi.org/10.1109/TMI.2004.836872

[31] Polman CH, Reingold SC, Edan G, Filippi M, Hartung HP, Kappos L, Metz LM, et al. Diagnostic criteria for multiple sclerosis: 2005 revisions to the McDonald criteria. Ann. Neurol. 2005; 58: 840-846. PMid:16283615 http://dx.doi.org/10.1002/ana.20703

[32] Shah M, Xiao Y, Subbanna N, Francis S, Arnold DL, Collins DL, Arbel T. Evaluating intensity normalization on MRIs of human brain with multiple sclerosis. Med. Imag. Anal. 2011; 15: 267-282. PMid:21233004 http://dx.doi.org/10.1016/j.media.2010.12.003

[33] Simmons A, Tofts PS, Barkers GJ, Arridge SR. Sources of inhomogeneity in spin echo images at 1.5 T. Magn. Reson. Med. 1994; 32: 21-128. http://dx.doi.org/10.1002/mrm.1910320117

[34] Thompson AJ, Hobart JC. Multiple sclerosis: assessment of disability and disability scales. J. Neur. 1998; $254(4): 189-196$. http://dx.doi.org/10.1007/s004150050204

[35] Wang L, Lai H-M, Barker GJ, Miller DH, Tofts PS. Correction for variations in MRI scanner sensitivity in brain studies with histogram matching. Magn. Res. Medic. 1998; 39(2): 322-327. PMid:9469718 
[36] Wang Z, Bovik A, Sheikh H, Simoncelli E. 2004; Image quality assessment: From error measurement to structural similarity; IEEE Trans. Imag. Proces. 2008; 13(4): 600-612. http://dx.doi.org/10.1109/TIP.2003.819861

[37] Weszka JS, Dyer CR, Rosenfield A. A comparative study of texture measures for terrain classification. IEEE Trans. Syst., Man. Cybern. 1976; SMC(6): 269-285.

[38] Wu CM, Chen YC, Hsieh K-S. Texture features for classification of ultrasonic images. IEEE Trans. Med. Imag. 1992; 11: 141-152. PMid:18218367

[39] Zhang J, Tong L, Wang L, Lib L. Texture analysis of multiple sclerosis: A comparative study; Magn. Res. Imag. 2008; 26(8): 1160-1166. PMid:18513908 http://dx.doi.org/10.1016/j.mri.2008.01.016 